\title{
Ground tissue circuitry regulates organ complexity in cereal roots
}

\section{Carlos Ortiz-Ramírez ${ }^{1,2}$, Poliana Coqueiro Dias Araujoㄹ, Sanqiang Zhang ${ }^{1}$, Edgar Demesa- Arevalo $^{3}$, Zhe Yan ${ }^{4,5}$, Xiosa Xü ${ }^{3}$, Ramin Rahni ${ }^{1}$, Thomas R. Gingeras ${ }^{3}$, David Jackson ${ }^{3}$, Kimberly L. Gallagher ${ }^{4}$, and Kenneth D. Birnbaum ${ }^{{ }^{*}}$}

\author{
${ }^{1}$ Center for Genomics and Systems Biology, Department of Biology, New York University, \\ New York, NY 10003, USA. \\ ${ }^{2}$ UGA Laboratorio Nacional de Genómica para la Biodiversidad, CINVESTAV Irapuato, \\ Guanajuato 36821, México. \\ ${ }^{3}$ Cold Spring Harbor Laboratory, Cold Spring Harbor, NY 11724, USA. \\ ${ }^{4}$ University of Pennsylvania, School of Arts and Sciences, Philadelphia, PA 1904, USA \\ ${ }^{5}$ Current address: The National Key Facility for Crop Gene Resources and Genetic Improvement \\ (NFCRI)/Key Lab of Germplasm Utilization (MOA), Institute of Crop Sciences, Chinese \\ Academy of Agricultural Sciences, Beijing 100081, China
}

*Correspondence to: ken.birnbaum@nyu.edu.

One sentence summary: Single-cell RNA-seq maps the maize root transcriptome uncovering a mechanism that regulates cortex layer number.

\begin{abstract}
:
Most plant roots have multiple cortex layers that make up the bulk of the organ and play key roles in physiology, such as flood tolerance and symbiosis. However, little is known about the formation of cortical layers outside of the highly reduced anatomy of the model Arabidopsis. Here we use single-cell RNAseq to rapidly generate a cell resolution map of the maize root, revealing an alternative configuration of the tissue formative SHORT-ROOT (SHR) signaling pathway adjacent to an expanded cortex. We show that maize SHR protein is hypermobile, moving at least eight cell layers into the cortex. Higher-order SHR mutants in both maize and Setaria have reduced numbers of cortical layers, showing that the SHR pathway controls expansion of cortical tissue in grasses that sets up anatomical complexity and a host of key traits.
\end{abstract}

\section{Main text}

Roots are radially symmetrical organs composed of three fundamental tissue types, the epidermis on the outside, the ground tissue at the middle, and a core of vascular elements plus pericycle that 
lie in a central cylinder known as the stele (1). The ground tissue is further divided into two different cell types, the endodermis and cortex, which are arranged as concentric layers around the stele. Variations in ground tissue patterning, particularly the number of cortex cell layers, are common across species and represent one of the defining features giving rise to interspecies root morphological diversity (1). This diversity allows plants to cope with biotic and abiotic stress and adapt to challenging environments. For example, roots with a multilayered cortex can develop mycorrhizal symbiotic associations, form specialized cortex-derived parenchyma for carbohydrate storage, and develop aerenchyma to avoid hypoxia in flood conditions (2-4). Therefore, an important ongoing question in root biology is how tissue patterning is adjusted to produce different root morphologies, and what are the underlying changes in the genetic networks controlling developmental differences in patterning among species.

A current limitation to answer this question is that our knowledge of radial patterning mechanisms in roots comes largely from the study of Arabidopsis, which possesses a simple root anatomy. In Arabidopsis, only two ground tissue layers develop in primary development, one endodermal and one cortical that originate from an asymmetrical cell division at or near the initials or stem cells (5). This division is controlled by the SHORT-ROOT (SHR)/SCARECROW (SCR) genetic pathway $(6,7)$. Mutants in either transcription factor develop a monolayered ground tissue. In addition, SHR mutants lack an endodermis, giving SHR a role in both tissue formation and cell identity (8).

Mechanistically, SHR functions as a mobile signal whose protein travels from the stele, where the gene is transcribed, into the surrounding endodermis, where it induces the expression of the downstream transcription factor $S C R$ (7). The pathway then triggers the division that generates the cortex and endodermis layers (8). It has been suggested that additional movement of SHR from the stele further out into the cortex could cause extra cell divisions, giving rise to multiple cortex layers (9). However, the role of the pathway in a species with multiple cortex layers has not been functionally tested, and its involvement in cortical expansion has been unclear. For example, in Cardamine hirsute, a close relative of $A$. thaliana, the formation of multiple cortex layers appears to be independent of the SHR-SCR pathway, instead controlled by the activity of HD-ZIPIII proteins (10). Thus, the genes that control the elaboration of multiple cortex layers remain a central question. In addition, it is not known to what degree different layers of the morphologically similar cortex tissue are specialized in their function. One barrier to answering these questions is that highresolution transcriptomics for comparative studies in species with multilayered cortex are lacking.

To address this gap, we first sought to produce a high-resolution spatial and temporal map of gene expression in a complex root that could provide clues to the genetic networks controlling morphological diversity in patterning. Therefore, we generated cell type-specific gene expression profiles using high-throughput single cell RNAseq (scRNAseq) to profile maize roots. Maize is a valuable model for comparative studies because its roots develop multilayered cortical tissues (89 cortex cell layers) within the root meristem, and it is amenable to protoplast generation, an essential step in plant scRNAseq (11). However, a major challenge of scRNAseq studies in species for which genomic resources are limited is the correct identification of cell types. Preliminary analysis showed that using homologs of Arabidopsis markers obtained by high-throughput cell sorting, did not provide a clear identification of morphologically homologous cell types in maize. This is likely because gene orthology is not always apparent and localization over such broad evolutionary distance is not well conserved. An alternative resource -- an extensive set of tissuespecific markers for cell sorting, which was used to generate cell-identity marker lines in Arabidopsis -- was not available in maize. 
To overcome this problem, we first took advantage of the concentric arrangement of tissues in roots to develop a technique to fluorescently mark cell layers by dye penetrance (Dye Penetrance Labeling, DPL). In brief, a highly permeable dye (Syto 40 - blue) stains the entire root with low but detectable staining in stele (Fig. 1A), whereas a weakly permeable dye (Syto 81 - green) stains the outer tissue layers strongly, with a gradual drop in signal intensity towards the inner tissues (Fig.1A). This dual labeling was highly reproducible across roots and batches, allowing us to use the blue/green ratio gradient among the concentric layers of the root to separate inner from outer cell types using Fluorescence Activated Cell Sorting (FACS). We calibrated dye ratio to radial position by using DPL on a line expressing a fluorescent protein driven by the SCR promoter (ZmSCR1::RFP; Fig. 1B), which expresses in the endodermis, a middle layer of the root. RFP positive cells were used to calibrate a reference dye ratio for this middle layer, allowing demarcation of inner and outer tissues (Fig. S1A). We dissected root tips (5 mm from tip) and then rapidly digested their cell walls, sorting cells belonging to different tissue layers using their specific dye ratio. We also generated a set of whole meristem protoplasts vs intact root controls to filter out any effects of cell wall digestion. Importantly, digested and undigested controls clustered closely together and replicate samples yielded highly consistent profiles (Fig. S1B,C). To validate the DPL dataset, we compared our epidermal and stele layer sorts to a previous study that used mechanical separation of inner vs outer layers (12) and found an $80 \%$ agreement (Fig. 1D,E). In this manner, we developed a set of at least 170 markers for each radially arranged tissue (Fig. S1D). In addition, we obtained expression profiles of the root cap by dissection and quiescent center (QC), using FACS on a stable QC marker line, ZmWOX5::tagRFPt (Fig.S1E).

To generate a single cell resolution map of the maize root meristem, we then dissected root tips from 7 day old wild-type B73 maize seedlings and enzymatically digested their cell walls, as above. We then used the cells to prepare single cell cDNA libraries using the 10x Genomics Chromium platform. A total of 4,324 high quality cells were sequenced in three different batches with a median of 15,254 UMIs/cell and 3,929 detected genes/cell. A total of 17 cell clusters were defined and visualized in two dimensions in Seurat using the uniform manifold approximation and projection (UMAP) method (13). To quantify cell identity and classify clusters using the DPL markers, we applied the Index of Cell Identity (ICI) algorithm (14), which generates a cell identity score based on the mean expression of a predefined marker gene set, in this case, from FACS isolated tissues (Fig. 2A \& Fig. S2). Overall, the technique allowed us to identify, with high confidence, 16 of the 17 UMAP clusters, providing a detailed spatial map of transcripts in highly specific tissues of the maize root (Fig 2B).

The high-resolution cellular map of the meristem showed multiple cell type subclusters within the stele and, interestingly, within the cortex as well, suggesting cellular specialization within that tissue's multiple layers. However, because root cells differentiate as they transition away from the root tip, the possibility remained that some subclusters merely represented different maturation states of a single cell type. To distinguish groups formed by distinct identity rather than differentiation state, we further generated a set of cell maturation marker transcripts by dissecting 16 longitudinal root slices that together comprised the meristematic, transition, and elongation zones, and subjected the samples to RNAseq analysis (Fig. 2 C). Using K-means clustering, we identified three main expression programs: early differentiation transcripts (high expression on the meristem and gradual decrease towards the transition zone), transition zone transcripts (specific to the mid-maturation point), and late differentiation transcripts (low expression in the meristematic zone and gradual increase towards the elongation zone). We then generated a cellular 
differentiation score to label the differentiation status of each cell, resolving developmental trajectories of cells in our high-resolution map of cell identities (Fig. 2 D).

We found that, in a few cases, the state of cell maturation is indeed the main factor influencing grouping of cells into subclusters. Six clusters had the same identity as immediately adjacent clusters but representing a different state of maturation. However, the majority of subclusters were composed of cells with a wide range of differentiation states, showing that the grouping in most cases represented distinct cell identities. Importantly, while one cortex subcluster appeared to be a precursor state of mature cortex (cluster 1), our analysis confirmed the existence of at least four distinct cortex subtypes (Fig. 2B, clusters 2,1,14,15). Furthermore, using the ROC algorithm in Seurat, which identified 1,395 differentially expressed genes (DEGs) across all clusters, we found 446 transcripts that mark some subset of the four different cortical subtypes (Fig. 2E). Thus, we provide quantitative evidence for the sub specialization of cortex that underlies expansion of root complexity, providing strong evidence for cortical cell diversification.

A key question is what signaling mechanisms allow maize to form the extra layers that permit cortex sub specialization. We observed that a short list of functional markers with a role in patterning or cell identity in Arabidopsis had conserved localization in homologous tissues in maize (e.g., SUC2 (phloem), MYB46 (xylem), RSL1 (epidermis), and LBD29 (stele)). However, the localization of the core patterning gene $S H R$ was surprising, as single cell data showed its expression was specific to the endodermis and not to the stele (Fig. 2E), where the Arabidopsis ortholog is expressed (8). All three maize paralogs of SHR (designated ZmSHR1, ZmSHR2, and ZmSHR2-h) showed the same endodermal enrichment in the profiles generated by single-cell and DPL/reporter analysis (Fig. 3A, B). We speculated that the expression of this mobile, division inducing transcription factor adjacent to the cortex could be related to a role in the expansion of that tissue.

To confirm SHR transcript localization, we used $1.4 \mathrm{~kb}$ upstream and downstream regions of ZmSHR2 to drive the expression of a nuclear-localized TagRFPt. Confocal images of root longitudinal sections from ZmSHR1::TagRFPt lines showed signal in the root endodermis in agreement with our dye sorted and single cell profiles (Fig. 3A,B,C). Notably, no signal was found in the stele, confirming that SHR2 transcript localizes to different cell types in maize compared to Arabidopsis. Furthermore, given evidence that rice SHR proteins are hypermobile when expressed heterologously in Arabidopsis (8), we assessed whether maize SHR protein could move from the endodermis, where it is expressed, into the adjacent cortex. For this, we made a natively expressed protein reporter of ZmSHR1 fused to YFP (ZmSHR1::SHR1-YFP). Indeed, compared to endodermal localization of the SHR1 found by dye sorting and single-cell RNA-seq, the maize SHR1 protein reporter was present in the cortex (Fig. 3D). Moreover, SHR1-YFP signal was not restricted to the immediately adjacent tissue layer as in Arabidopsis but was observed in all cortex layers, suggesting that the endogenous maize SHR1 protein moves through at least 8 cortex layers (Fig. 3D).

SHR's role in promoting division in Arabidopsis works through direct interaction with SCR (15). Therefore, we also generated maize SCR reporter lines to determine colocalization with $S H R$. As noted in our DPL calibration, nuclear localized RFP expressed from the ZmSCR promoter revealed a clear signal in the root endodermis, showing SCR transcript localization is conserved in Arabidopsis and maize (Fig. 3E). However, natively expressed ZmSCR-GFP protein fusions showed a strong signal in the stele, suggesting that SCR protein in maize moves from cell-to-cell in the opposite direction to SHR (Fig. 3F). This shows that SHR and SCR colocalize only in the 
endodermis and not in the extra cortical layers, while SCR protein has an additional domain in the stele. A SCR translational reporter in a second monocot, Setaria viridis (Setaria), showed the same localization in the stele, further corroborating the divergent localization of SCR protein in monocots and suggesting SHR functions independently of SCR in the cortex (Fig. S3).

The model that implicates SHR in cortical expansion posits that increased outward movement of the protein could trigger periclinal cell divisions giving rise to extra ground tissue layers (9). To test the model, we targeted the three different maize SHR paralogs to generate loss-of-function mutants in maize using CRISPR-Cas9. We recovered mutants in two of the genes (ZmSHR2 and ZmSHR2-h), including the most highly expressed paralog. Single mutants in Zmshr2 and Zmshr2$h$ had no apparent difference in their root anatomy compared to wild type siblings. However, Zmshr2/2-h double mutants showed a significant reduction in the number of cortical layers: with most roots having 7 layers compared to 8 to 9 layers in wild type (Fig 4A,B,C,D). Mutants in the single SHR gene in Arabidopsis lack an endodermal layer. However, staining for suberin and morphological analysis showed that Zmshr2/2-h roots still developed an endodermis (Fig. S4). We posit that the remaining functional $Z m S H R 1$ gene in the Zmshr2/2-h background may still enable specification of endodermal identity. Alternatively, ZmSCR1 (and ZmSCR1-h) may be the primary factors in the specification of the maize endodermis (16). Overall, our results suggest that SHR function in maize is necessary for the full expansion of cortical identity.

We sought to validate the monocot SHR phenotype with a more severe loss of function by testing its role in Setaria viridis, a close relative of maize. In Setaria, we were able to generate loss of function mutants in the two SHR orthologs using CRISPR-Cas9 (Fig. S5). One Setaria SHR mutant, Svshr2, showed a slight reduction in cortical layers, while a single mutant in the second, Svshr1, showed no phenotype. However, double mutants showed a dramatic reduction in the number of ground tissue layers: 1-2 layers compared to 4-5 layers in wild type siblings (Fig. 4E, $\mathrm{F}, \mathrm{G} \& \mathrm{H}$ ). These results corroborate the role of $S H R$ in controlling the expansion of cortical layers in two monocots.

We note that the extra cortical divisions mediated by SHR could function through direct interaction with SCR by mediating successive divisions of the cortex-endodermal split near the stem cell niche, where the two proteins overlapped in maize. Alternatively, SHR hypermobility could lead to divisions directly in the cortical layers independently of direct interaction with SCR. At present, we cannot distinguish between these two models.

Notably, the results show that SHR has a role in monocots controlling the expansion of cortex, which sets up many key traits for environmental acclimation. This illustrates how a divergent role of a key patterning gene leads to a major difference in organ patterning. Furthermore, the results show that rapid transcriptome mapping using single cell dissection can provide insights into the mechanisms that mediate anatomical diversity. The use of dye labeling to generate a scaffold locational map together with scRNAseq now provides a detailed maize root tissue map that can be used as a reference map in monocots.

\section{References}

1. K. Esau, Anatomy of seed plants. (Wiley, New York, ed. 2d, 1977), pp. 550 
2. A. H. Gunawardena, D. M. Pearce, M. B. Jackson, C. R. Hawes, D. E. Evans, Characterisation of programmed cell death during aerenchyma formation induced by ethylene or hypoxia in roots of maize (Zea mays L.). Planta 212, 205-214 (2001).

3. T. Yamauchi, T. D. Colmer, O. Pedersen, M. Nakazono, Regulation of Root Traits for Internal Aeration and Tolerance to Soil Waterlogging-Flooding Stress. Plant Physiol 176, 1118-1130 (2018).

4. K. K. Fugate et al., Colocalization of sucrose synthase expression and sucrose storage in the sugarbeet taproot indicates a potential role for sucrose catabolism in sucrose accumulation. Journal of plant physiology 240, 153016 (2019).

5. L. Dolan et al., Cellular organisation of the Arabidopsis thaliana root. Development 119, 71-84 (1993).

6. P. N. Benfey et al., Root development in Arabidopsis: four mutants with dramatically altered root morphogenesis. Development 119, 57-70 (1993).

7. K. Nakajima, G. Sena, T. Nawy, P. N. Benfey, Intercellular movement of the putative transcription factor SHR in root patterning. Nature 413, 307-311 (2001).

8. Y. Helariutta et al., The SHORT-ROOT gene controls radial patterning of the Arabidopsis root through radial signaling. Cell 101, 555-567 (2000).

9. S. Wu et al., A plausible mechanism, based upon Short-Root movement, for regulating the number of cortex cell layers in roots. Proc Natl Acad Sci U S A 111, 16184-16189 (2014).

10. G. Di Ruocco et al., Differential spatial distribution of miR165/6 determines variability in plant root anatomy. Development 145, (2018).

11. A. Senn, P.-E. Pilet, Isolation and some Morphological Properties of Maize Root Protoplasts. Zeitschrift für Pflanzenphysiologie 100, 299-310 (1980).

12. N. Opitz et al., Extensive tissue-specific transcriptomic plasticity in maize primary roots upon water deficit. J Exp Bot 67, 1095-1107 (2016).

13. T. Stuart et al., Comprehensive Integration of Single-Cell Data. Cell 177, 1888-1902 e1821 (2019).

14. I. Efroni, P. L. Ip, T. Nawy, A. Mello, K. D. Birnbaum, Quantification of cell identity from single-cell gene expression profiles. Genome Biol 16, 9 (2015).

15. H. Cui et al., An evolutionarily conserved mechanism delimiting SHR movement defines a single layer of endodermis in plants. Science 316, 421-425 (2007).

16. T. E. Hughes, O. V. Sedelnikova, H. Wu, P. W. Becraft, J. A. Langdale, Redundant SCARECROW genes pattern distinct cell layers in roots and leaves of maize. Development 146, (2019).

17. C. Ortiz-Ramirez, E. D. Arevalo, X. Xu, D. P. Jackson, K. D. Birnbaum, An Efficient Cell Sorting Protocol for Maize Protoplasts. Curr Protoc Plant Biol 3, e20072 (2018).

18. K. M. Crawford, P. C. Zambryski, Subcellular localization determines the availability of non-targeted proteins to plasmodesmatal transport. Curr Biol 10, 1032-1040 (2000).

19. Y. Lei et al., CRISPR-P: a web tool for synthetic single-guide RNA design of CRISPR-system in plants. Mol Plant 7, 1494-1496 (2014).

20. S. N. Char et al., An Agrobacterium-delivered CRISPR/Cas9 system for high-frequency targeted mutagenesis in maize. Plant Biotechnol J 15, 257-268 (2017). 
21. T. P. Brutnell et al., Setaria viridis: a model for C4 photosynthesis. Plant Cell 22, 25372544 (2010).

\section{Acknowledgments}

Funding: K.D.B and D.J. are supported by NSF grant IOS-1934388. K.D.B, D.J. and T.R.G. were supported by NSF grant IOS 1445025. K.D.B. is funded by NIH grant R35GM136362. D.J. is funded by NSF IOS-1930101. K.L.G NSF PGRP-23020. Author contributions: C.O.R. performed all transcriptomic experiments, expression analysis, and mutant analysis. C.O.R. and K.D.B. designed the experiments and wrote the manuscript. C.O.R., K.D.B., D.J., T.R.G., and K.L.G conceived the project and guided the experiments. P.C.D.A. and S.Z. assisted in mutant analysis and marker analysis. E.D.A. and X.X. generated transcriptional reporters in maize. Z.Y. generated the translational reporters in maize and the Setaria mutants. R.R. designed and carried out microscopy protocols. Data materials and availability: All raw and processed/normalized expression data is available through the Gene Expression Omnibus under the SuperSeries accession GSE172302.

\section{List of Supplementary materials:}

Materials and Methods

Fig. S1. FACS sorting of RPF positive and dye-stained protoplasts and clustering analyses of tissue transcriptomes.

Fig. S2. ICI analysis of UMAP defined clusters. Heatmaps show ICI analysis of each cell (columns) in each cluster.

Fig. S3. SCR protein is located in the stele in Setaria viridis roots.

Fig. S4. Endodermal identity analysis by suberin staining in wild type and Zmshr1/3 in maize.

Fig. S5. CRSIPR-Cas9 gene editing in ZmSHR and SvSHR genes caused frameshift mutations. 
A
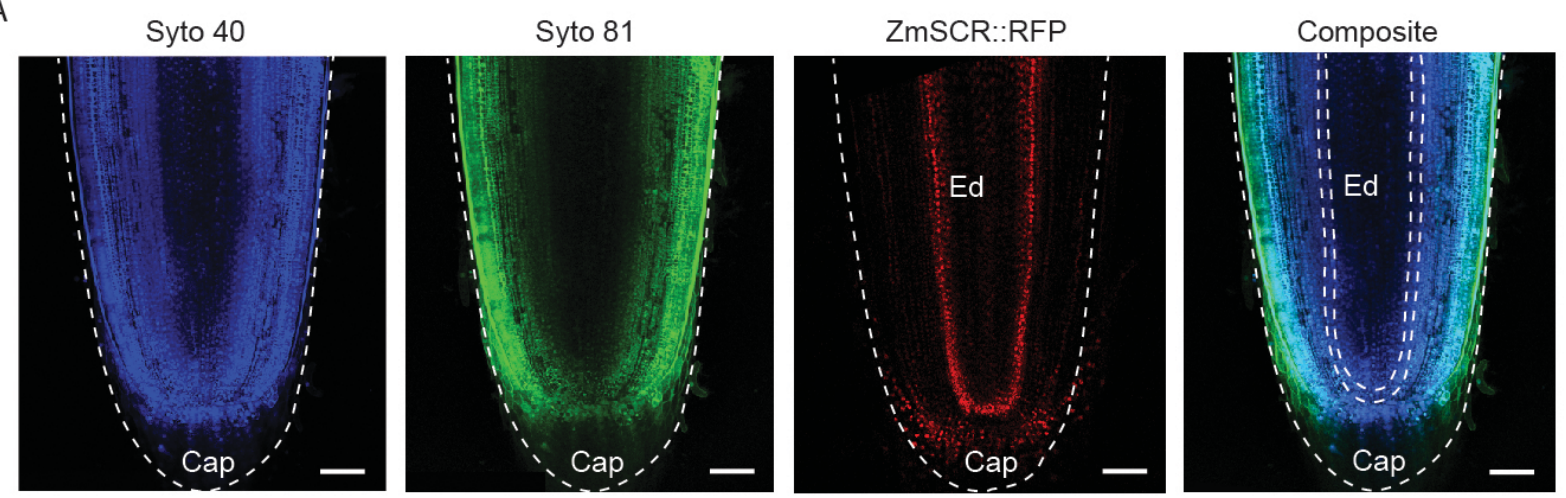

B
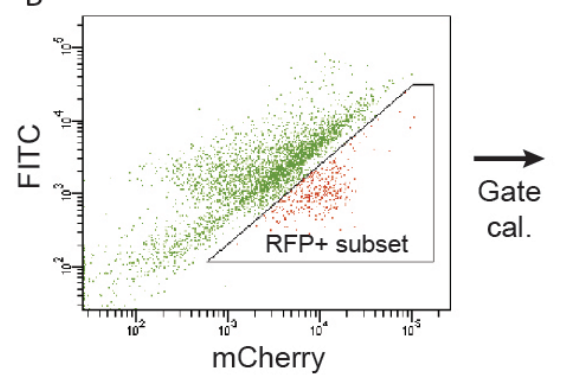

C

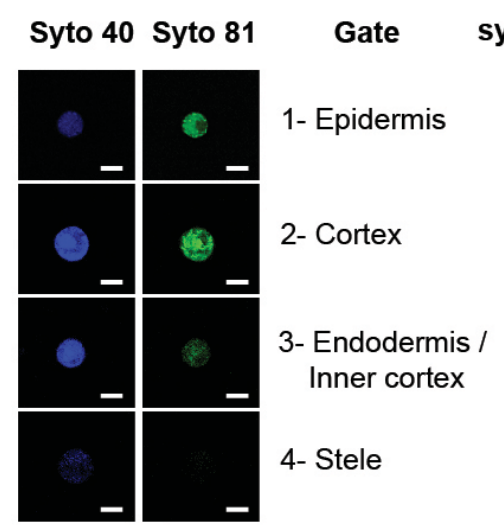

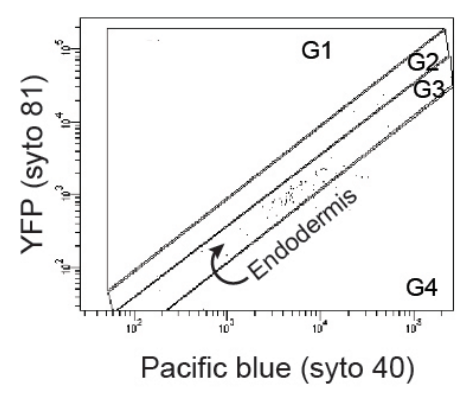

D

syto $40 / 81$

0.92

2.12

6.7

28.9
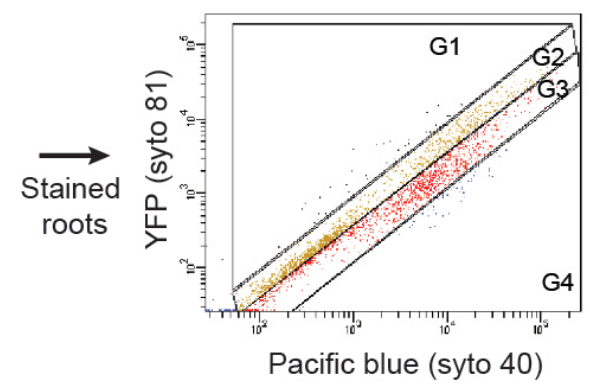

E

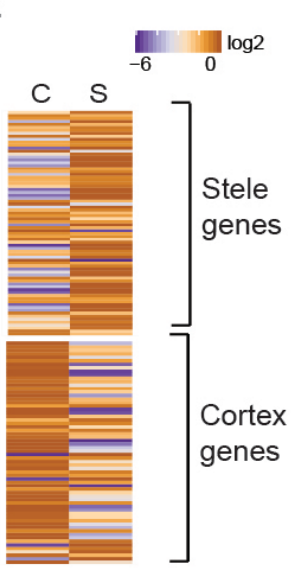

Fig. 1. Dye Penetrance Labeling (DPL) and tissue transcriptome analysis in maize.

(A) Representative images of a deeply penetrating dye (Syto 40), a superficially penetrating dye (Syto 81), the ZmSCR::tagRFPt marking endodermis, and a composite image of Syto 40 and Syto 81 staining, showing position of the endodermal (Ed) layers in dashed region. (B) Cell sorting gating strategy, showing the ZmSCR::tagRFPt population (left), backgated onto a YFP vs Pacific Blue plot with RFP positive (second from left), and (third from left) the gated boundaries for endodermal, outside of endodermis (G1,G2), and inside of endodermis (G4). (C) Validation of ratio metric cell sorting strategy by collecting sorted cells from gates and quantifying fluorescence from microscopy images. (D) Validation of sorted cell RNA-seq profiles via analysis of known markers. (E) Global validation comparing sorted cells vs. mechanically dissected stele and cortex 
bioRxiv preprint doi: https://doi.org/10.1101/2021.04.28.441892; this version posted April 29, 2021. The copyright holder for this preprint (which

was not certified by peer review) is the author/funder, who has granted bioRxiv a license to display the preprint in perpetuity. It is made available under aCC-BY-NC-ND 4.0 International license.

tissues, with heat map showing expression in sorted cortex vs. stele gates, categorized by previously determined stele and cortex markers. Scale bars are $100 \mu \mathrm{m}$ in (A) and $15 \mu \mathrm{m}$ in (C). 
bioRxiv preprint doi: https://doi.org/10.1101/2021.04.28.441892; this version posted April 29, 2021. The copyright holder for this preprint (which was not certified by peer review) is the author/funder, who has granted bioRxiv a license to display the preprint in perpetuity. It is made available under aCC-BY-NC-ND 4.0 International license.

A
UMAP
Cluster 5
$\mathrm{ICI}$ score
Normalized $\mathrm{ICl}$ (cutoff)
Assign identity

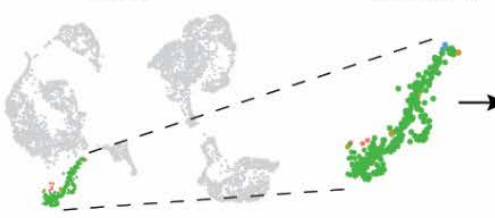

$\underline{\bar{U}}$
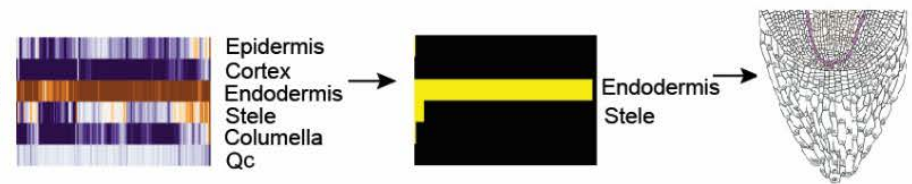

B

C
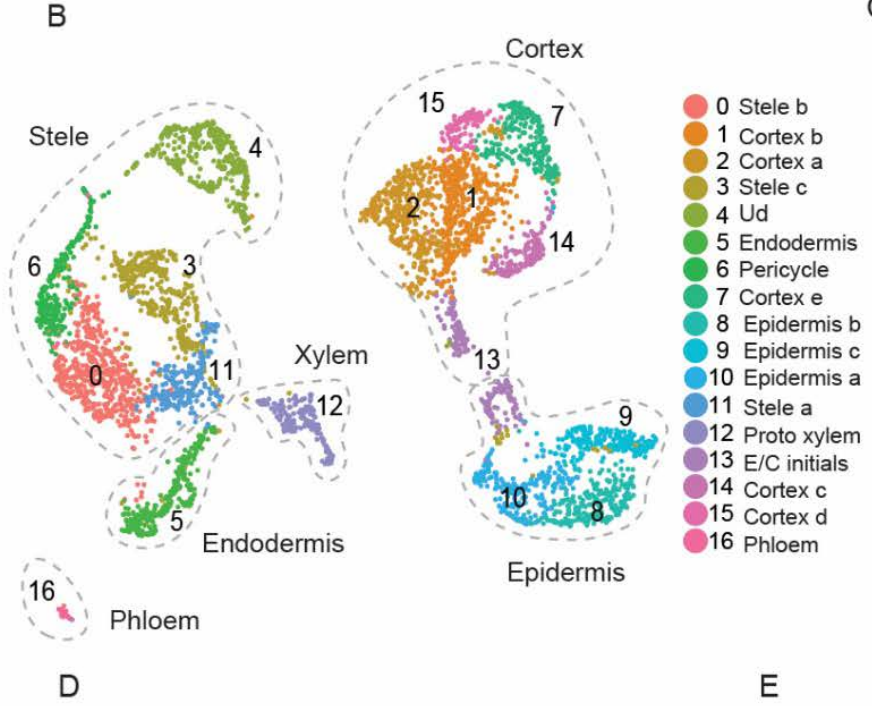

Epidermis

16 Phloem

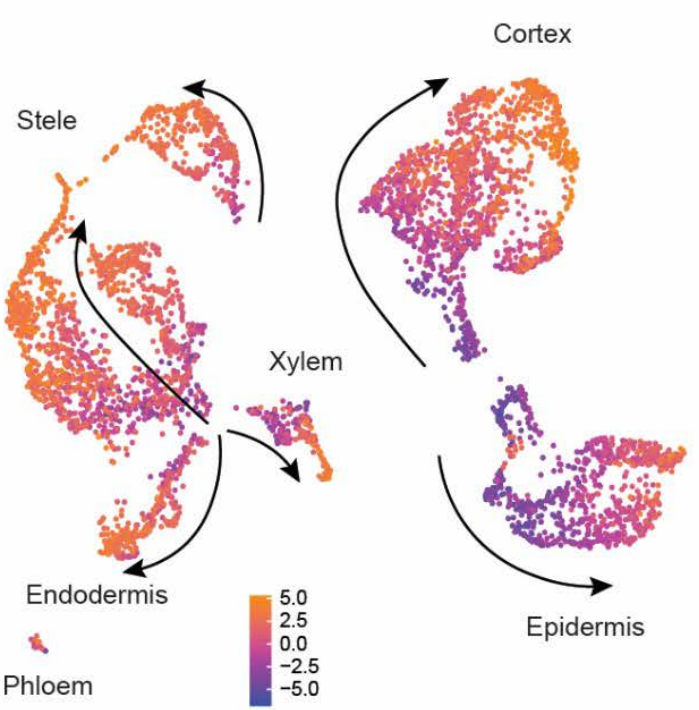

E
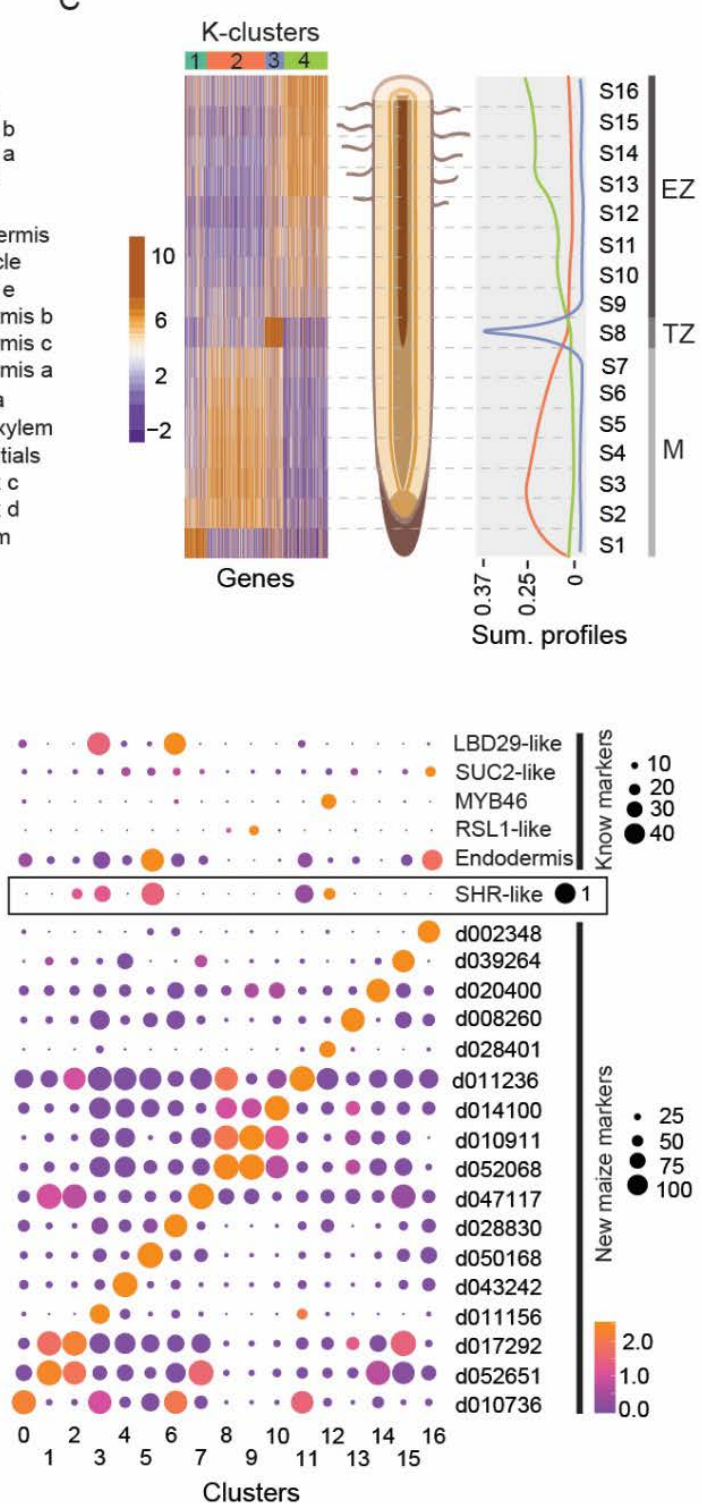

Figure 2. Single-cell RNA-seq spatial and temporal transcriptome maps of the maize meristem. 
(A) The ICI method of examining cells from a specific cluster in the UMAP analysis (e.g., Cluster 5), scoring the identity of each cell using tissue-specific markers obtained from DPL in Fig. 1, normalizing the scores by false discovery rate (see Methods). (B) Cluster identities determined by rough mapping (using ICI) and fine mapping using cell-specific markers. (C) Heat map of highly variant genes along a longitudinal axis of the root meristem, using thin sections of the root. Development patterns show transcripts/markers that peak in the early meristem (M), transition zone (TZ), and elongation zone (EZ). (D) Trajectories of developmental "pseudo-time" in each cell cluster mapped onto the same UMAP depicted in B, where a differentiation score is calculated as a log2 ratio of all EZ/M markers identified in C. (E) Validation of single-cell RNA-seq cluster calls with known markers (top). New markers for each cluster are shown at the bottom. 

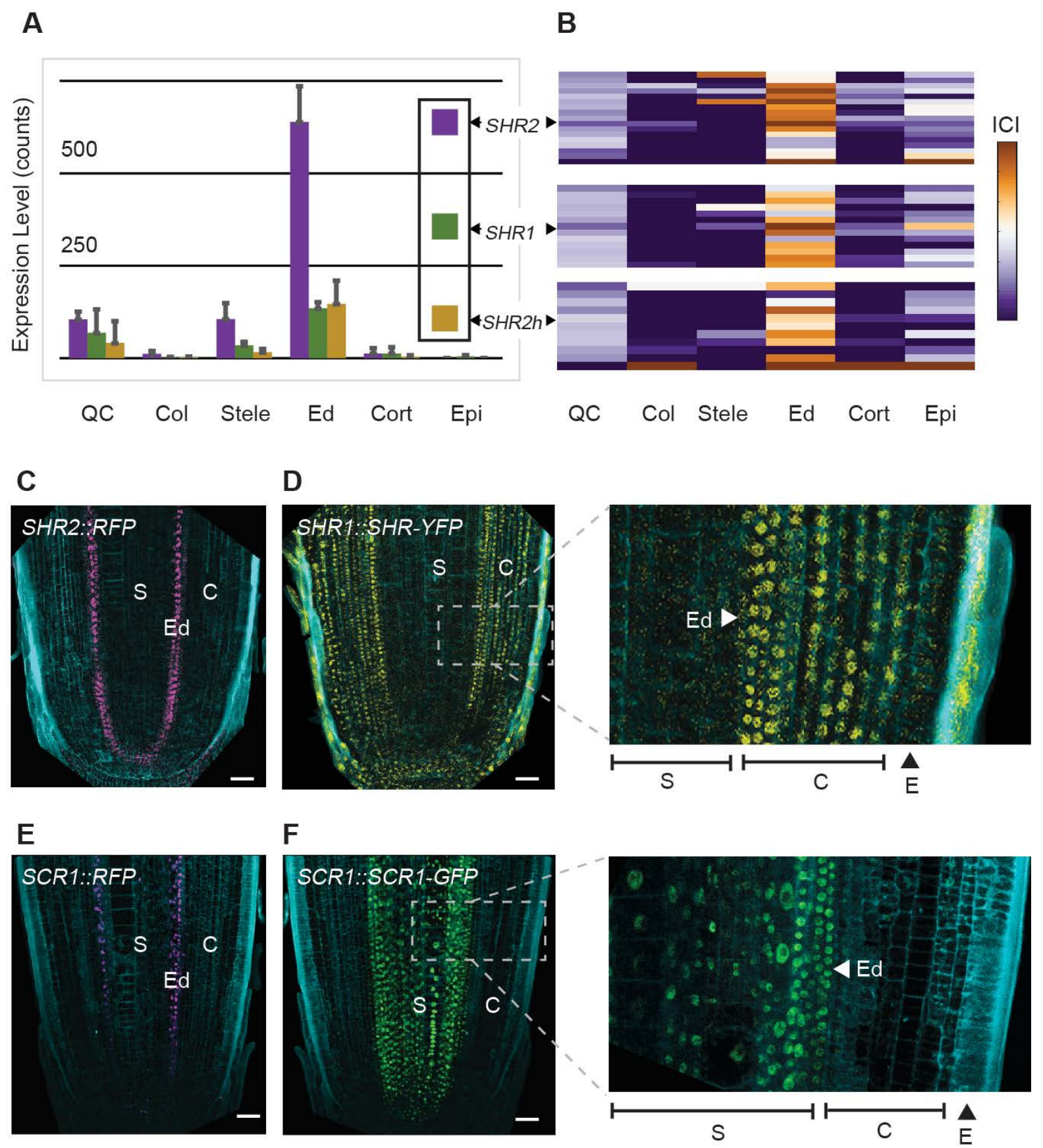

Figure 3. SHR and SCR expression in maize endodermis and differences between transcriptional and translational reporters.

(A) Expression of the three ZmSHR paralogs (SHR1,2,2-h) as detected in sorted cells. Error bars are std based on three replicates. (B) ICI analysis of cells expressing either of the three SHR paralogs, identifying the SHR-expressing cells as having endodermal identity. (C) ZmSHR2 transcriptional reporter. (D) ZmSHR1 translational reporter, with inset showing enlarged view of boxed area and expression in all cell layers of cortex. (E) Transcriptional reporter for ZmSCR1. 
bioRxiv preprint doi: https://doi.org/10.1101/2021.04.28.441892; this version posted April 29, 2021. The copyright holder for this preprint (which

was not certified by peer review) is the author/funder, who has granted bioRxiv a license to display the preprint in perpetuity. It is made available under aCC-BY-NC-ND 4.0 International license.

(F) Translational reporter for ZmSCR1 with inset showing enlarged view of boxed area and expression in many layers of the stele. Scale bars are $50 \mu \mathrm{m}$. 
A

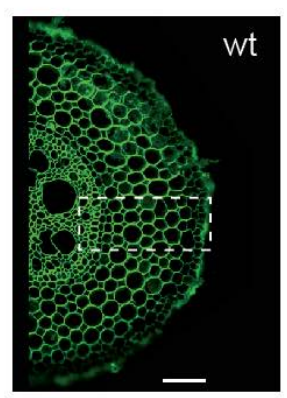

E

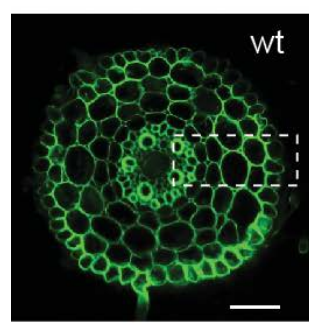

B

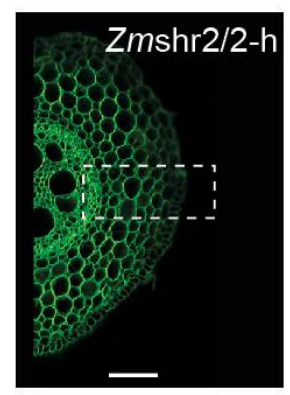

$\mathrm{F}$

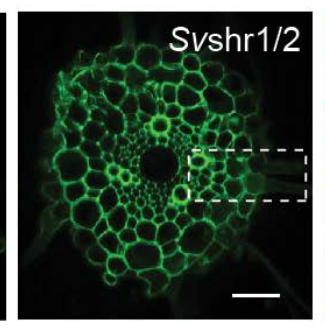

C

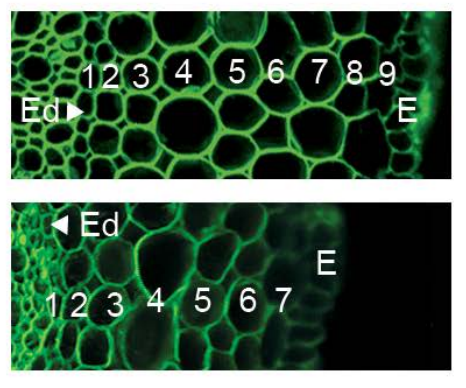

G

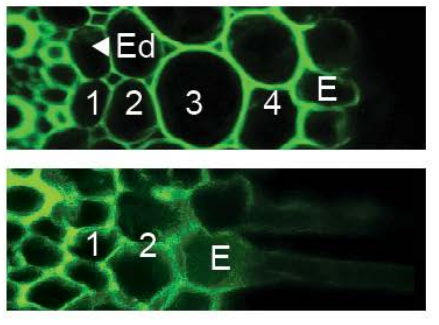

D

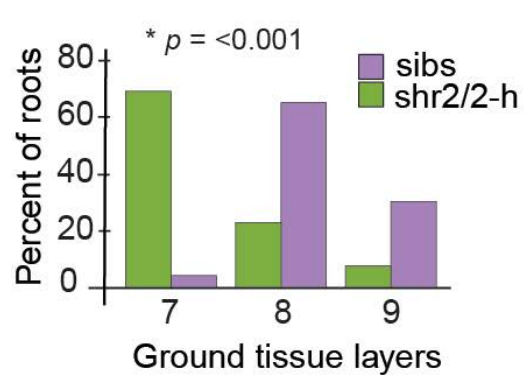

H

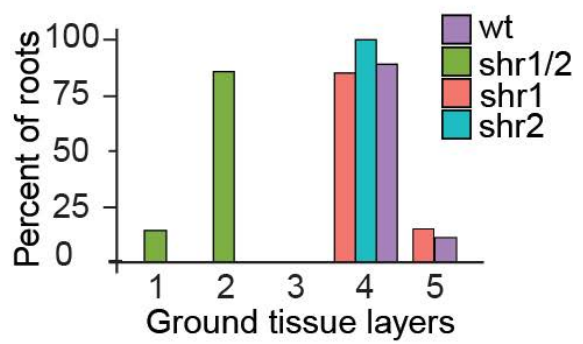

Fig. 4. Cortical cell layer analysis in wild type and shr mutants in monocots.

(A,B) Representative maize root cross-sections of wild type in A vs. Zmshr2/2-h double homozygous mutant in B. (C) Enlarged and labelled regions from dashed boxes in A and B showing stelar (S), endodermal (Ed), and cortical layers of wild type (top) and Zmshr2/2-h mutant (bottom). (D) Quantification of the cortical cell layers in wild type and het sibs (n=23) vs. Zmshr2/2-h mutant (n=13). $P$ value was estimated with Mann-Whitney Rank Sum Test (E,F) Representative cross sections of Setaria wild type in E and Svshr1/2 mutants in F. (G) Enlarged and labelled regions from dashed boxes in $\mathrm{E}$ and $\mathrm{F}$ showing stelar (S), endodermal (Ed), and cortical layers of wild type (top) and Svshr1/2 mutant (bottom). (H) Quantification of cortical layers in Setaria wild type $(n=9)$, Svshr1 single $(n=7)$, Svshr2 single $(n=6)$ and double mutants $(\mathrm{n}=7)$. Scale bars are $100 \mu \mathrm{m}$ in (A), (C), and $50 \mu \mathrm{m}$ in (E), (F). 


\section{Materials and Methods}

\section{Accession numbers maize}

SHR:

Zm00001d029607 (ZmSHR1)

Zm00001d021973 (ZmSHR2)

Zm00001d006721 (ZmSHR2-h)

SCR:

Zm00001d052380 (ZmSCR1)

Zm00001d005029 (ZmSCR2)

WOX5:

Zm00001d042821 (ZmWOX5B)

\section{Accession numbers Setaria}

SHR

Sevir.9G361300 (SvSHR1)

Sevir2G383300 (SvSHR2)

SCR

Sevir8G008100 (SvSCR1)

\section{Plant growth Conditions}

For protoplast generation and collection of root slices, maize seeds were incubated in 3\% (v/v) sodium hypochlorite for $8 \mathrm{~min}$. Seeds were then transferred to a sheet of wetted heavy-weight germination paper, rolled and covered with aluminum foil to prevent roots from exposure to direct light. Rolled paper was placed in a 2-gallon plastic container and kept inside a Percival high light chamber for 7 days with a dark-light cycle of $16 \mathrm{hr}$ light at $27^{\circ} \mathrm{C}$ and $8 \mathrm{hr}$ dark at $24^{\circ} \mathrm{C}$ and $50 \%$ humidity (17). For plant propgation and crosses, maize plants were grown inside a greenhouse with controlled light and temperature under a $16 \mathrm{hr}$ light at $28^{\circ} \mathrm{C}$ and $8 \mathrm{hr}$ dark at $22^{\circ} \mathrm{C}$ for 3 months. Setaria seeds were sterilized as described before and germinated on square plates containing $0.5 \mathrm{X}$ Murashige and Skoog (MS) medium. Plates were kept in a Percival high light chamber for 14 days with a dark-light cycle of $16 \mathrm{hr}$ light at $27^{\circ} \mathrm{C}$ and $8 \mathrm{hr}$ dark at $24^{\circ} \mathrm{C}$ and $50 \%$ humidity until collection of root tissue for microscopic analyses. Setaria plants used for seed bulking and genetic crosses were grown in a walk-in chamber exposed to the same light/dark cycle and temperature as described above.

\section{Generation of maize reporter lines}

ZmSHR2, ZmSCR1 and ZmWOX5 transcriptional reporter lines were constructed using the Multisite GATEWAY (Invitrogen) recombination system using the pTF101 plasmid as a destination vector, all regulatory sequences were driving the expression of TagRFPt protein fused 
to four copies of the Nuclear Localization Signal (NLS) of VirD2 from Agrobacterium tumefasciens (18). For ZmSHR2, 1,405 bp of the upstream region and 1,445 bp of the downstream region were cloned into the reporter construct. For ZmSCR1, 1,704 bp of the upstream sequence and 2,054 bp of the downstream sequence were cloned into the construct. Finally, for ZmWOX5, 3,705 bp of upstream sequence and 2,826 bp of downstream sequence were cloned into the reporter. All expression plasmids were transformed in Agrobacterium tumefasciens strain EHA101 for maize transformation. At least two independent lines were used to verify the expression patterns.

For the ZmSHR translational fusion, the ZmSHR promoter (3,118 bp upstream from zmSHR), CDS and YFP were cloned and assembled into pTF101.1 destination vector via BamHI+NcoI, NcoI+SbfI and SbfI+AflII cloning site respectively. For the ZmSCR translational fusion, the ZmSCR promoter (3,261 bp upstream from zmSCR), CDS and GFP were cloned and assembled into the pTF101.1 destination vector via BamHI+BlpI, BlpI+SbfI and SbfI+EcoRI cloning site respectively. All expression plasmids were transformed into Agrobacterium tumefasciens strain EHA101 and maize transformation was performed at the Plant Transformation Facility of Iowa State University.

\section{CRISPR-Cas9 mediated gene editing of maize SHR genes}

Null alleles for ZmSHR2 and ZmSHR2-h were generated using the CRISPR-Cas9 system. Five sgRNAs were designed to simultaneously target both SHR genes using CRISPR-P 2.0 webtool(19) based on B73 reference genome (GCAACACGTTCTGCACGCAG, GTCGAAGGACGTGTTCCGGT,

GCATTTCCACTCGCACGGCG, GCACTCCAGCAGCAGCTGCG). The array of sgRNAs under the ZmU6 and OzU6 promoters were synthesized and cloned into a pGW-Cas9 construct and transformed into the maize Hi-II accession using Agrobacterium tumefasciens (20). Transformed plants were screened by PCR amplification of the genomic region containing the targeted site. Transformed plants were backcrossed once to B73 and then plants with edited alleles were crossed reciprocally to segregate both ZmSHR2 and ZmSHR2-h edited alleles under the same genetic background. All phenotypic assays were done between plants harboring null alleles and their segregating siblings.

\section{SCR translational fusion in Setaria}

For SvSCR translational fusion, SvSCR promoter (2 kb upstream)+5UTR, and SviSCR CDS were assembled into modified PENTR 3C-YFP vector via SbfI+BamHI and BamHI+EcoRI cloning site, respectively. pSvSCR-SvSCR-YFP was cloned into modified PANIC 10A-Gateway destination vector using GATEWAY (Invitrogen) recombination system.

\section{CRISPR-Cas9 mediated editing of Setaria SHR genes}

sgRNA were designed using CRISPR-P (svSHR1: CGTGGCCGAACGACGCCCAC; svSHR2: TCTGCTAGAGTGCGCTCGGG). The sgRNA of svSHR1 was assembled into pJG338 and the 
sgRNA of svSHR2 was assembled into pJG340 via the BaeI cloning site. The vectors pJG471 containing Ubi1::TaCas9 and pJG338 or pJG340 were assembled into the pRLG103 destination vector via the AarI cloning site. Stable Setaria viridis transformation with CRISPR-Cas9 vectors was performed as described previously (21). T0 and T1 plants were screened by PCR targeting CRISPR editing site of svSHR1 or svSHR2.

\section{Dye Penetrance Labeling (DPL)}

Roots tips (5mm) from 7-day-old plantlets were placed on a tissue culture dish containing a solution of Syto $40(1 \mu \mathrm{M})$ and Syto $80(0.6 \mu \mathrm{M})$ in $3 \mathrm{ml}$ of $\mathrm{diH}_{2} \mathrm{O}$ containing L-cysteine $(2.5 \mathrm{mM})$ for $40 \mathrm{~min}$, shaking continuously at $60 \mathrm{rpm}$. Stained roots were washed at least 5 times in $\operatorname{diH}_{2} \mathrm{O}$ and transferred to protoplast generating solution (see below). In preliminary work, variation in staining was minimal from root to root, and modifying incubation time only changed fluorescent intensity but did not modified penetrance of either dye. Using the ratio of Syto40 to Syto 80 rather than absolute signal of either normalized for root-to-root variation.

\section{Protoplast Generation}

Protoplasts were generated from primary and seminal roots as described previously (17). In brief, roots were cut above the meristem at approximately 4 to $5 \mathrm{~mm}$ from the root tip and placed in pretreatment solution containing L-cysteine for $40 \mathrm{~min}(0.75 \mathrm{~g}$ sorbitol, $62 \mu 1$ of $1 \mathrm{M}$ L-cysteine in $25 \mathrm{ml}$ ) to improve enzyme efficiency and cell wall digestion. When root tips were used for DPL, the pretreatment solution also contained Syto 40 and Syto 81 dyes and without sorbitol, as it decreases dye penetrance efficiency. Cell walls were digested for $90 \mathrm{~min}$ in an enzyme solution optimized for maize roots [1.2\% cellulase “Onozuka” RS, 1.2\% cellulase “Onozuka” R10, 0.4\% macerozyme R-10 (all three Yakult Pharmaceutical Industry CO.), 0.36\% pectolyase Y-23 (MP Biomedicals)]. Protoplast were then filtered through a $40 \mu \mathrm{m}$ cell strainer and transferred to microcentrifuge tubes for centrifugation (3 min at $500 \mathrm{x}$ g). Protoplast pellets were washed and resuspended in washing solution (0.4 M mannitol, 0.02 M MES, 0.02 M KCl, 0.01 M CaCl, 0.015 mM BSA) and used immediately for fluorescence-activated cell sorting or single-cell RNAseq.

\section{Cell Sorting and Tissue Profiles}

A gating strategy was set up to delineate four gates, representing epidermis, cortex, endodermis and stele. First, we stained ZmSCR::RFP line using the DPL technique outlined above with Syto 81 and Syto 40. Protoplasts generated after staining were used to first identify RFP positive cells in a FITC vs. mCherry set of panels on a FACSAria II (Becton Dickinson). Those same cells were also visualized (backgated) in a YFP (for Syto 81) vs. Pacific Blue (for Syto 40) plot, allowing us to determine a gate that represented the narrow range of ratios that represented endodermal cells in YFP vs. Pacific Blue plot (G3). Using DPL and short digestion protocols that largely released epidermal protoplasts, we similarly determined the ratio range for epidermal cells (G1). The ratio range (Syto 80/Syto 40) between epidermal and endodermal cells was then labelled as the cortical layers (G2). The ratio range higher than the endodermal gate was labeled as stele (G4). A fifth 
sample representing the quiescent center (QC, with some xylem expression) was collected separately using a pWOX5::tagRFPt line and a similar gating strategy used before to detect the SCR::RFP line in the FITC vs. mCherry plot. For root cap isolation, we took advantage of the intrinsic properties of the cap, which is not amenable to enzymatic digestion. Root tips were thus digested with enzymatic solution for 90 min effectively removing cells of the root proper, and the remaining caps were inspected under a stereomicroscope and collected (30 caps for each replicate). All samples were subjected to the same RNA extraction and library preparation detailed below.

\section{Protoplast and Dye Staining Controls}

Controls were generated to assess the effects of protoplast generation: For protoplast generation, approximately 30 maize plants were germinated in germination paper and grown for 7 days as described above. Primary and seminal roots tips $(5 \mathrm{~mm})$ were harvested and split in half arbitrarily. Half of the roots were subjected to cell wall digestion as above, while the second half was processed for RNA extraction after manual dissection of the cap. Caps were removed to make the cell composition in each sample the same, since digested root preparations lack protoplasts from the cap.

\section{Root Slices}

Roots were grown on germination paper as described above and cross sections of primary roots about 80-100 $\mu \mathrm{m}$ thick were harvested at 7 days after germination in the following manner: three roots tips (10 mm long) were cut and placed on top of a microscope slide covered by a rectangular piece of germination paper. Root tips were aligned parallel to each other under a stereoscope, taking the tip of the roots as a refence. Once aligned, the germination paper was bent around and placed on top of the root tips covering the last $5 \mathrm{~mm}$ (proximal part of the root). A small balance weight (300g) was placed on top the germination paper to hold the roots in position while cutting the cross sections. Cross sections were cut using a feather microscalpel (Electron microscopy sciences, cat. 72045-30) starting from the tip of the root to the proximal region. 16 equivalent slices were obtained from two roots (replicates) and collected in individual tubes containing RTL buffer (Qiagen MicroRNAeasy Kit). Samples were flash frozen by placing liquid nitrogen in the tubes. RNA extraction and library preparation were done as described below.

\section{Small Sample RNA-seq}

RNA was extracted using the Qiagen MicroRNAeasy Kit. For dissected tissue samples (slices, protoplasting controls, and root cap), tissue samples were macerated in a $1.5 \mathrm{ml}$ Eppendorf filled with RLT buffer and ground by hand with a plastic pestle after flash freezing with liquid nitrogen. For sorted cells, the positive flow stream was sorted directly into RLT buffer, after which point the Qiagen MicroRNAeasy extraction protocol was performed, excluding tissue homogenization. We performed cDNA construction and library amplification with Nugen OvationRNA Amplification System V2 (Tecan). Libraries were made using the Nugen Ovation Ultralow DR Multiplex System (Tecan). DNA fragmentation was performed using the Covaris S220. Short read 
sequences were generated on an Illumina NextSeq 500 and were aligned using HISAT2 to the maize B73 v4 reference genome. Counts were normalized between samples using DESeq2's median of ratios method before analyzing for differentially expressed genes.

\section{Single cell RNA-seq}

Protoplasts were prepared as described above. An aliquot was stained with flourescein diacetate $(2 \mu \mathrm{g} / \mathrm{ml})$ for $3 \mathrm{~min}$ to determine viability before loading. Approximately 10,000 cells were loaded in a Single Cell A Chip (10x Genomics) per replicate. Three independent replicates were performed. Chips were loaded on a Chromium Controller (10x Genomics) to generate single-cell GEMs. Single-cell libraries were then prepared using the Chromium Single Cell 3' library kit, following manufacturer instructions. The quality of resulting DNA libraries was assessed with an Agilent TapeStation system. Library concentration was determined by quantitative PCR (qPCR) and sequenced with an Illumina NextSeq 550 platform using a 1x150 high-output configuration. Raw scRNAseq data was analyzed by Cell Ranger 2.2.0 (10x Genomics) to generate gene-cell matrices. Gene reads were aligned to maize B73 v4 reference genome.

\section{UMAP and ICI analysis}

Replicates (3 independently generated single-cell libraries) were integrated and cells mapped using the Seurat package v3.0 (13) as follows: first, genes with counts in fewer than three cells were excluded from the analysis and their counts were removed. Second, any cell with fewer than 200 total UMI were also removed. Next, counts were log-normalized and the 2000 most variable genes were identified for each replicate using the "vst" method in Seurat. Next, we used the FindIntegrationAnchors function to identify anchors between the three datasets, using 20 dimensions. A new profile with an integrated expression matrix containing cells from all replicates was produced with the IntegrateData function. For dimensionality reduction, the integrated expression matrix was scaled (linear transformed) using the ScaleData function, and Principal Component analysis (PCA) performed. The top 20 principal components were selected. Cells were clustered using a K-nearest neighbor (KNN) graph, which is based on the Euclidean distance in PCA space. The FindNeighbors and FindClusters function with a resolution of 0.6. was applied. Next, non-linear dimensional reduction was performed using the UMAP algorithm with the top 20 PCs.

For single cell ICI analysis, high quality cells were selected according to default cutoffs in CellRanger (10x Genomics). Each cluster was then analyzed using the Index of Cell Identity approach (14) using 50 markers for each of the six tissues isolated using the dye penetrance technique (4 tissues) and the two markers used to sort cells, ZmSCR::RFP and ZmWOX5::RFP. Markers were selected by correlation to tissue specific pattern including replicates (e.g., 11100

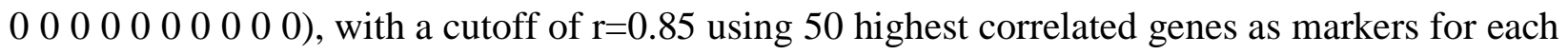
tissue. The False Discovery Rate was calculated by randomizing the marker by tissue type matrix 1000 times and using the $95^{\text {th }}$ percentile highest score for a given tissue as a cutoff for a 5\% FDR. 
The heatmap represents the $\log 2$ values of the observed ICI-95 ${ }^{\text {th }}$ percentile cutoff, which we termed the normalized ICI in Fig. 1. The ICI score for cells expressing SHR1, 2, or 2-h in Fig. 3 was calculated similarly.

\section{Microscopy for marker and mutant Analysis}

Quantification of ground tissue layers from wild type and mutant roots in maize and Setaria were performed by hand sectioning cross section "slices" of approximately $100 \mu \mathrm{m}$ acquired at approximately $10 \mathrm{~mm}$ from the tip. Cross sections were flipped onto the flat surface of a slide, mounted in water, and imaged using a Leica SPE confocal microscope. Samples were excited at $405 \mathrm{~nm}$ generating an autofluorescent profile that outlined cell walls collected at 505-555 nm. Maize and Setaria fluorescent reporter lines were imaged by performing longitudinal cuts of primary and seminal roots as close as possible to median. As above, root sections were mounted in water. The RFP markers were imaged with a $561 \mathrm{~nm}$ laser using an mCherry filter setting, while GFP and YFP markers were imaged with a 488 laser using FITC and YFP filter settings on the Leica SPE.

\section{Suberin Staining}

Anatomical characterization of wild type and mutant plants in maize was performed by first fixing roots in 50\% ethanol. Hand sectioned "slices" were sampled in the late differentiation zone just below the epicotyl junction. Root slices were stained with fluorol yellow 088 stain $\left(0.01 \% \mathrm{w} / \mathrm{v}\right.$, Sigma) in lactic acid (Sigma) in $70^{\circ} \mathrm{C}$ water bathing for 1 hour. The sections were then washed by deionized water and post-stained with $0.1 \%(\mathrm{w} / \mathrm{v})$ toluidine blue O (Fisher scientific) for $2 \mathrm{~min}$. 


\section{Supplemental Figures}

A

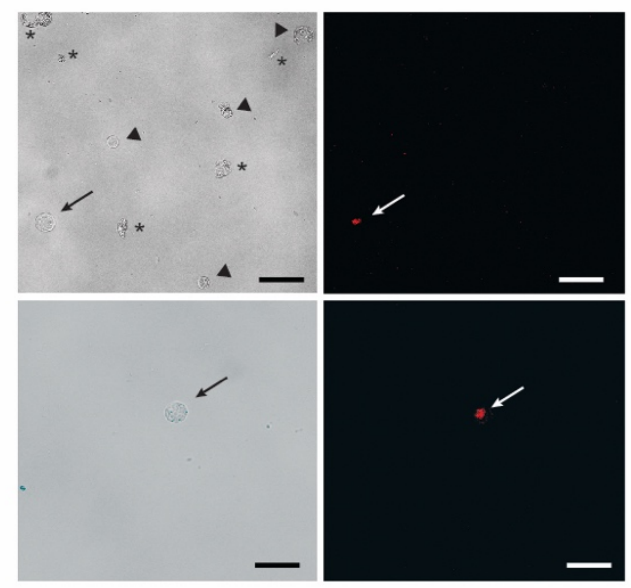

C

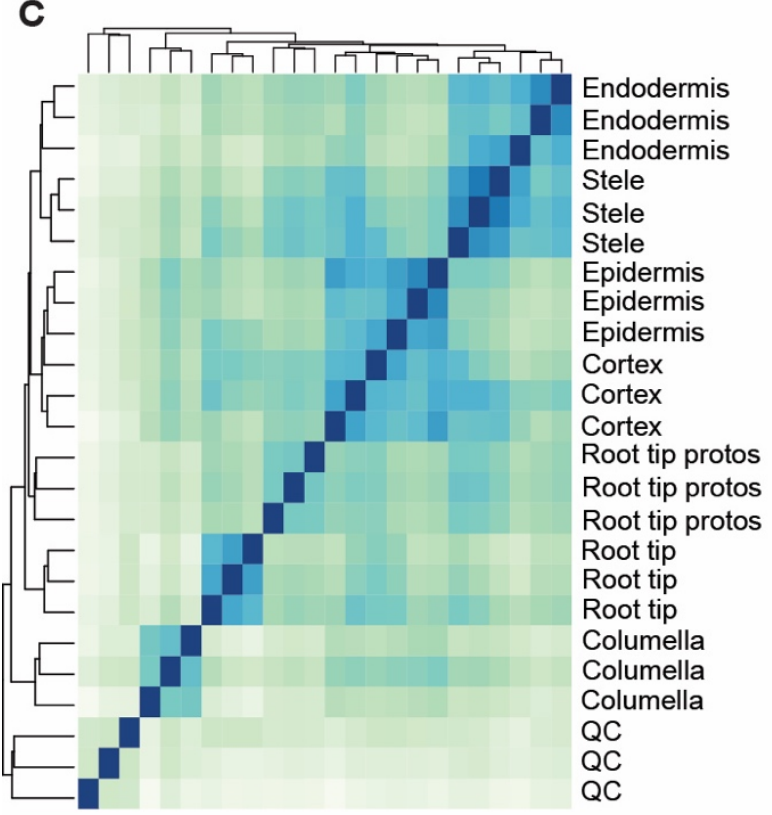

B

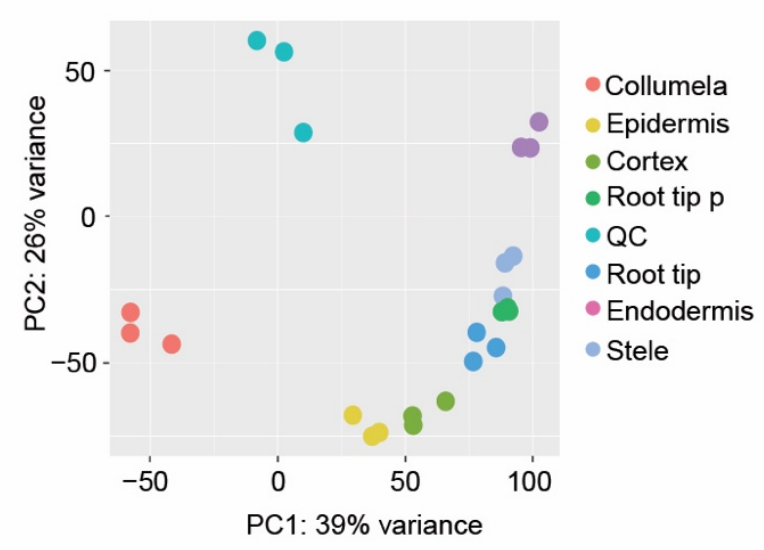

D

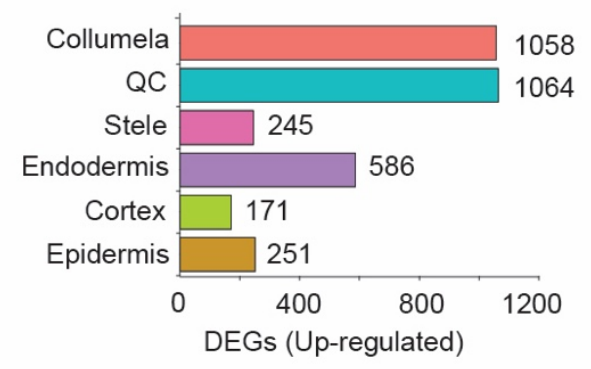

E

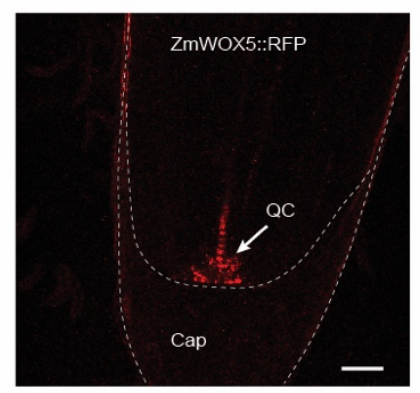

Fig. S1. FACS sorting of RPF positive and dye-stained protoplasts and clustering analyses of tissue transcriptomes.

(A) Bright field microscopy showing protoplasts (triangles) and debris (asterisks) from ZmSCR::tagRFP1 digested roots before (top left) and after (bottom left) FACS sorting. Fluorescence confocal microscopy confirmed that protoplasts expressing RFP were present (arrow, top right) and isolated (arrow, bottom right). (B) Principal Component Analysis (PCA) of 
tissue-specific gene expression patterns and whole roots vs. all root protoplast controls. (C) Hierarchical clustering dendrogram of tissues replicates showing sample similarity. (D) Number of upregulated differentially expressed genes. Root tip represents the undigested root meristem and root tip p/protos represents the same region of the root as a collection of protoplasts. Scale bars are $50 \mu \mathrm{m}$ in $\mathrm{A}$ and $100 \mu \mathrm{m}$ in $\mathrm{E}$. 
bioRxiv preprint doi: https://doi.org/10.1101/2021.04.28.441892; this version posted April 29, 2021. The copyright holder for this preprint (which was not certified by peer review) is the author/funder, who has granted bioRxiv a license to display the preprint in perpetuity. It is made available under aCC-BY-NC-ND 4.0 International license.

\section{Log2 fold over $5 \%$ Highest Randomization Value}

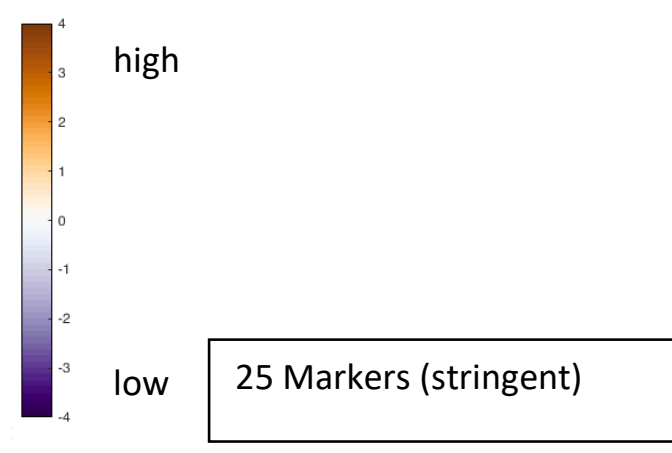

150 Markers
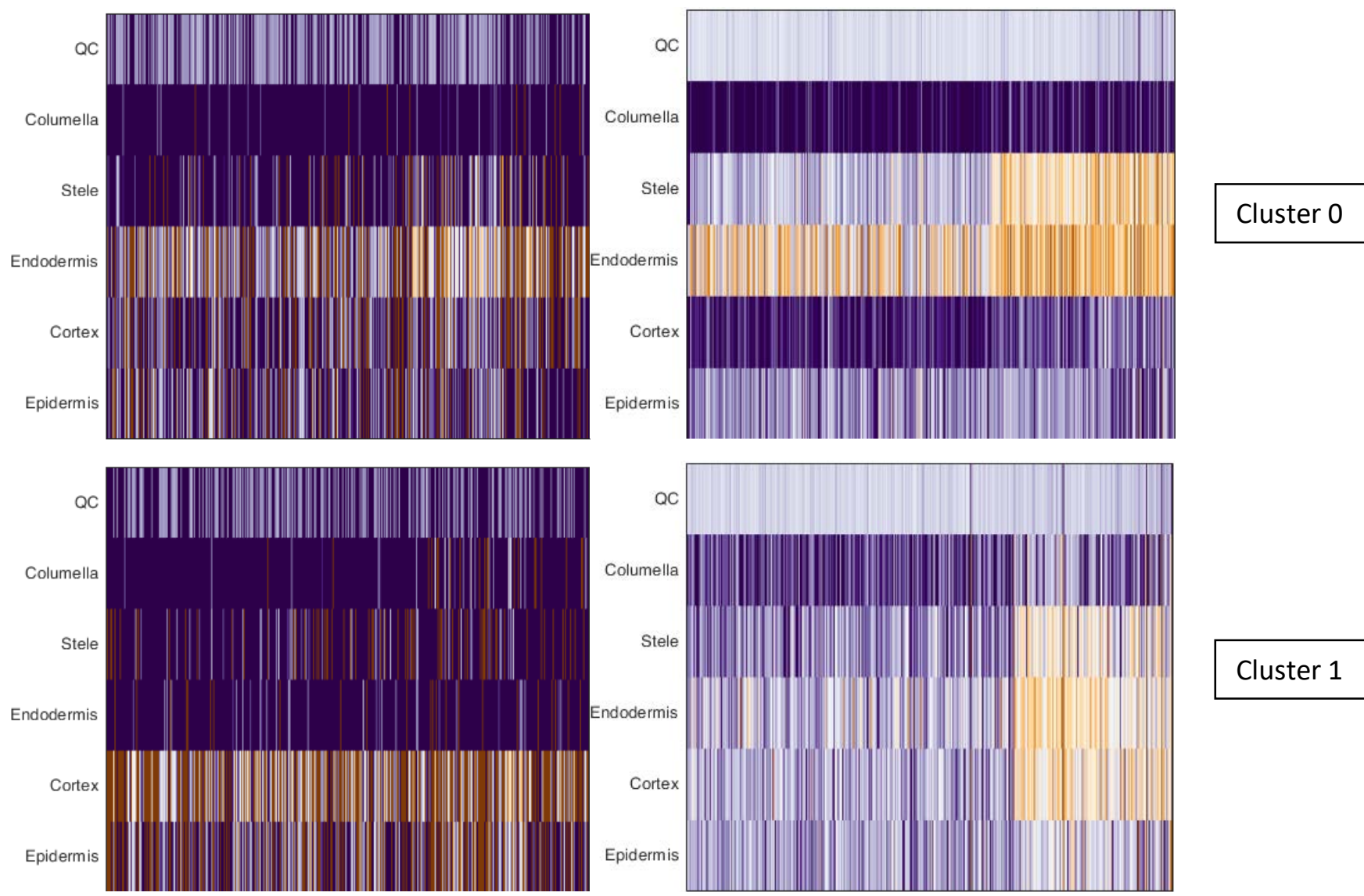

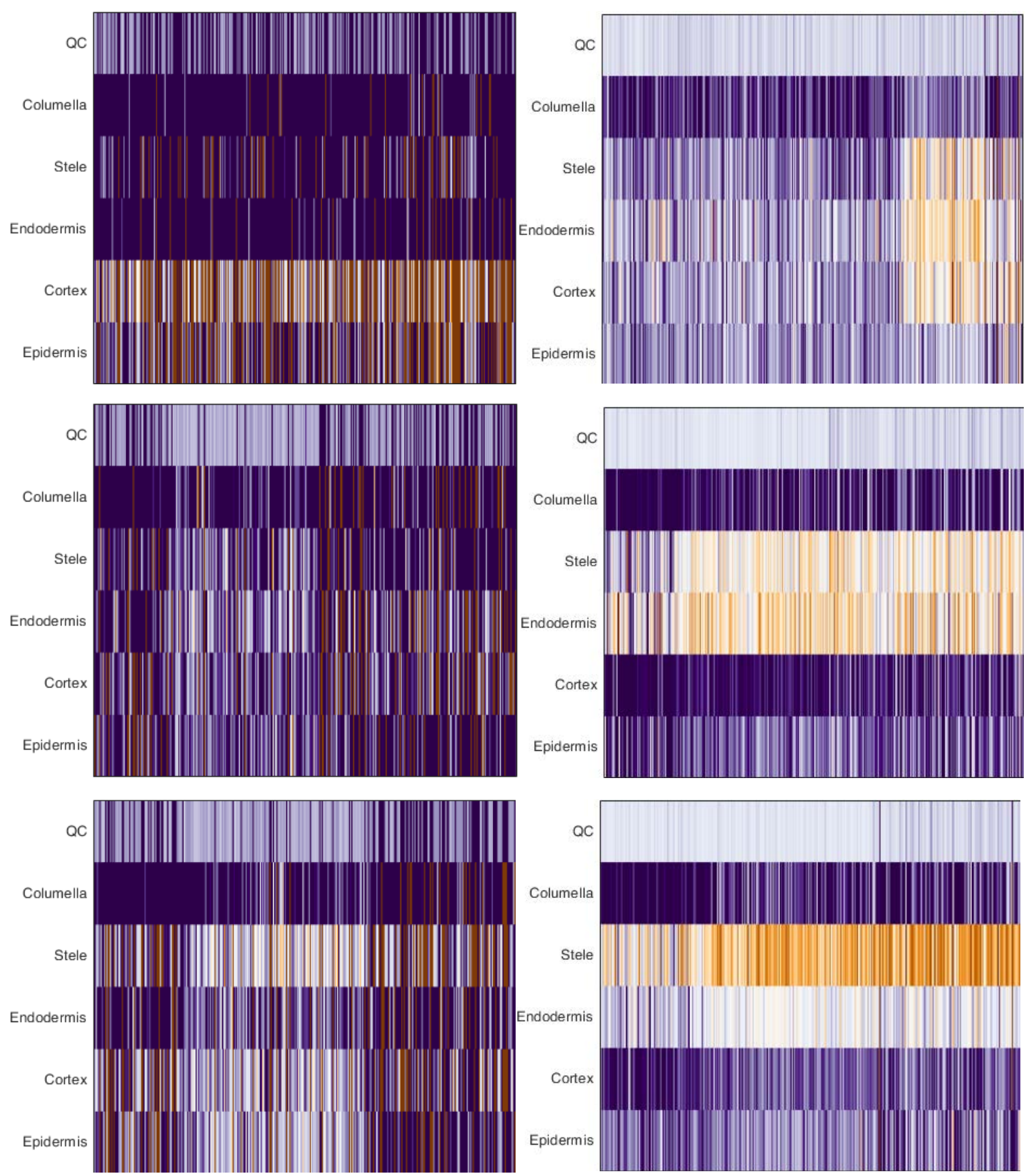

Cluster 2 

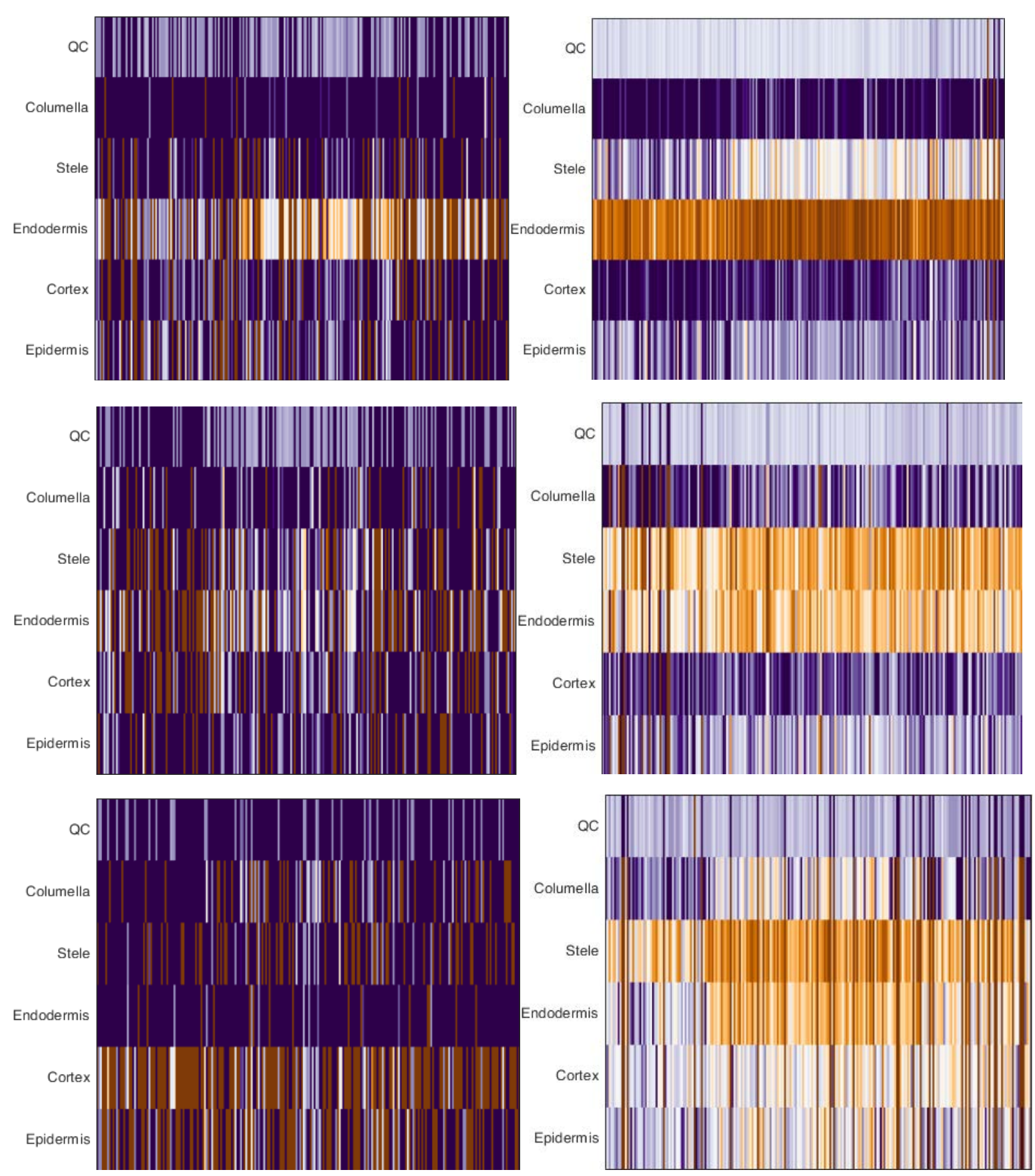

\section{Cluster 5}

Cluster 6 

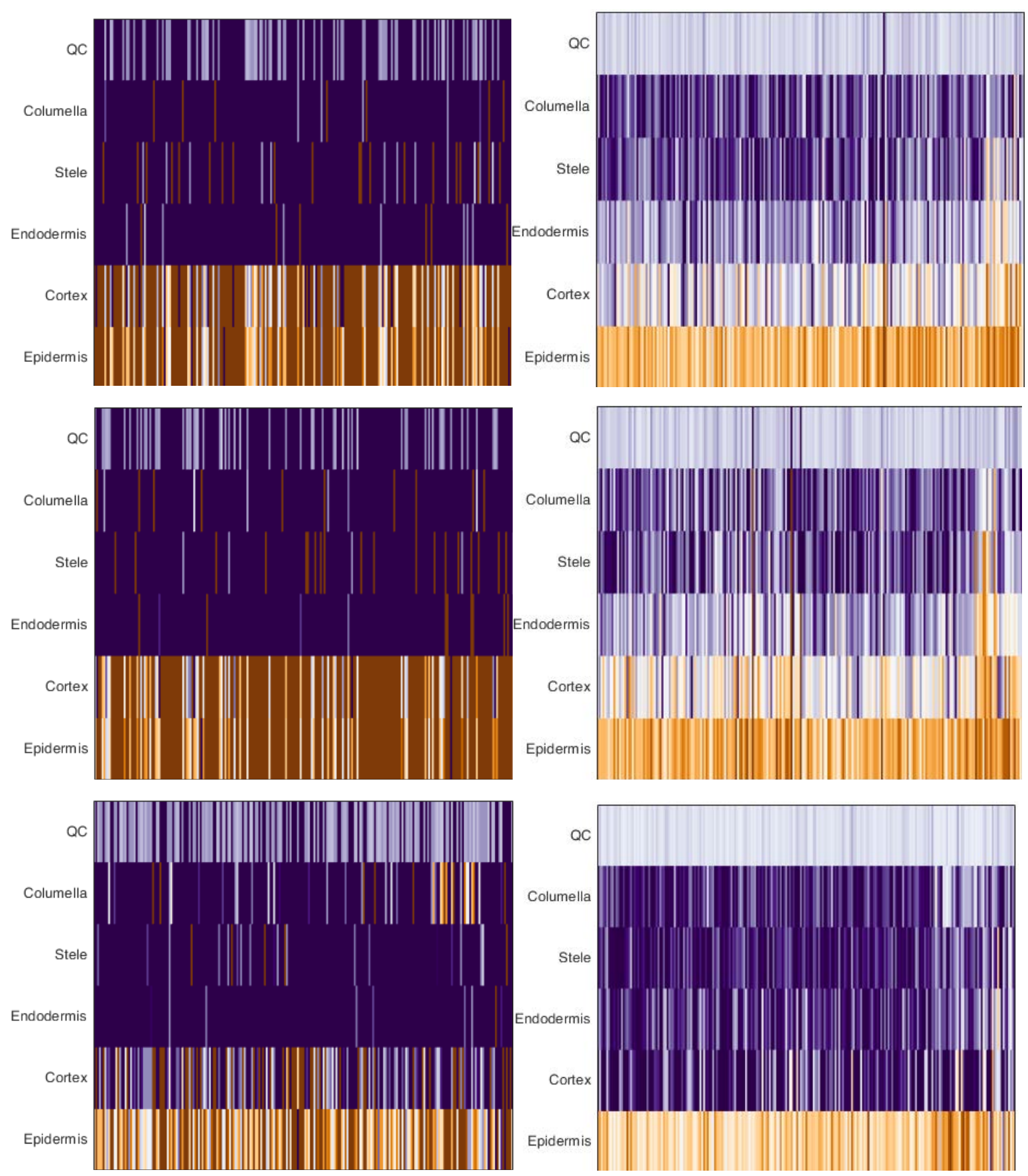

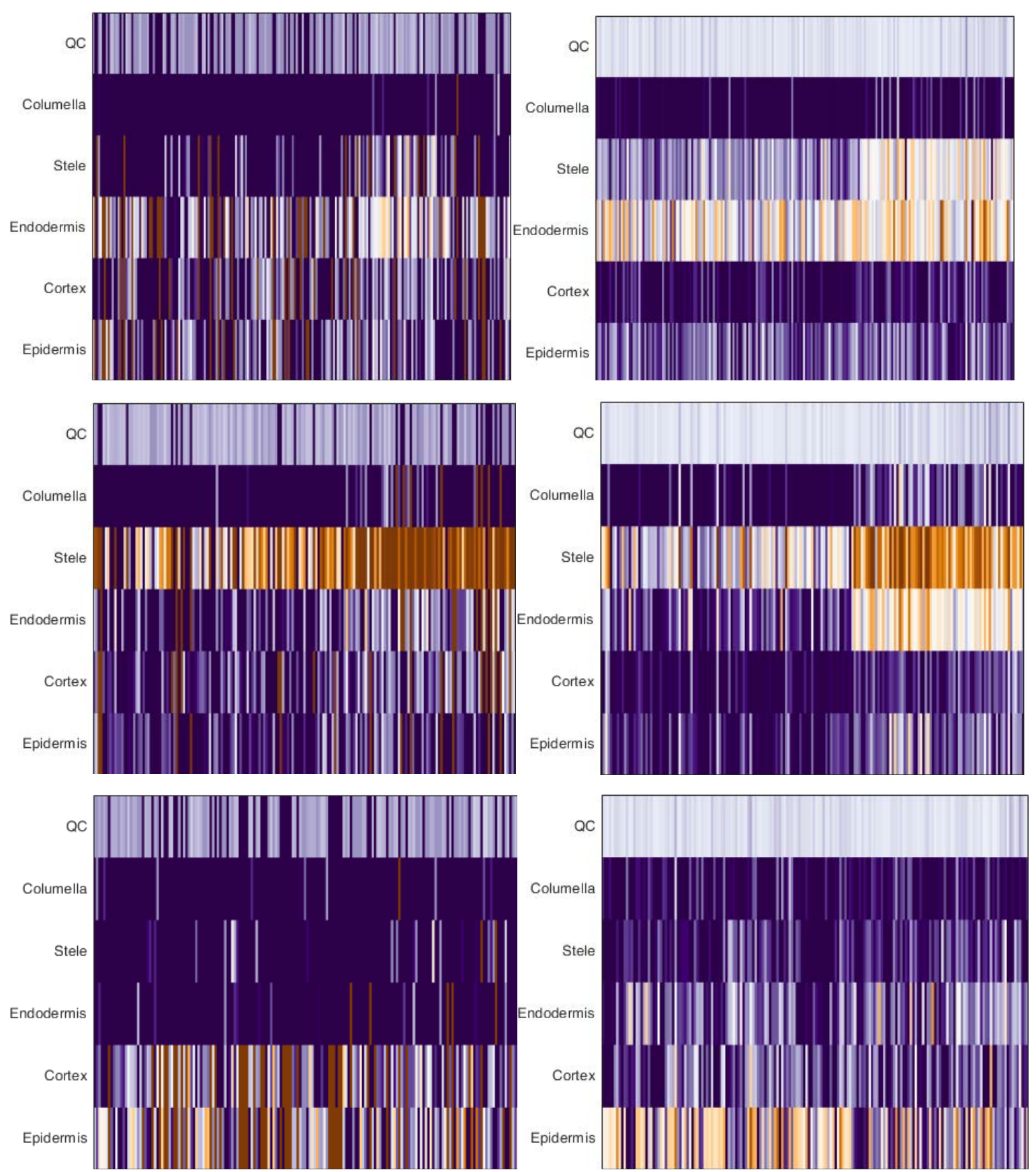

Cluster 11

Cluster 12

Cluster 13 

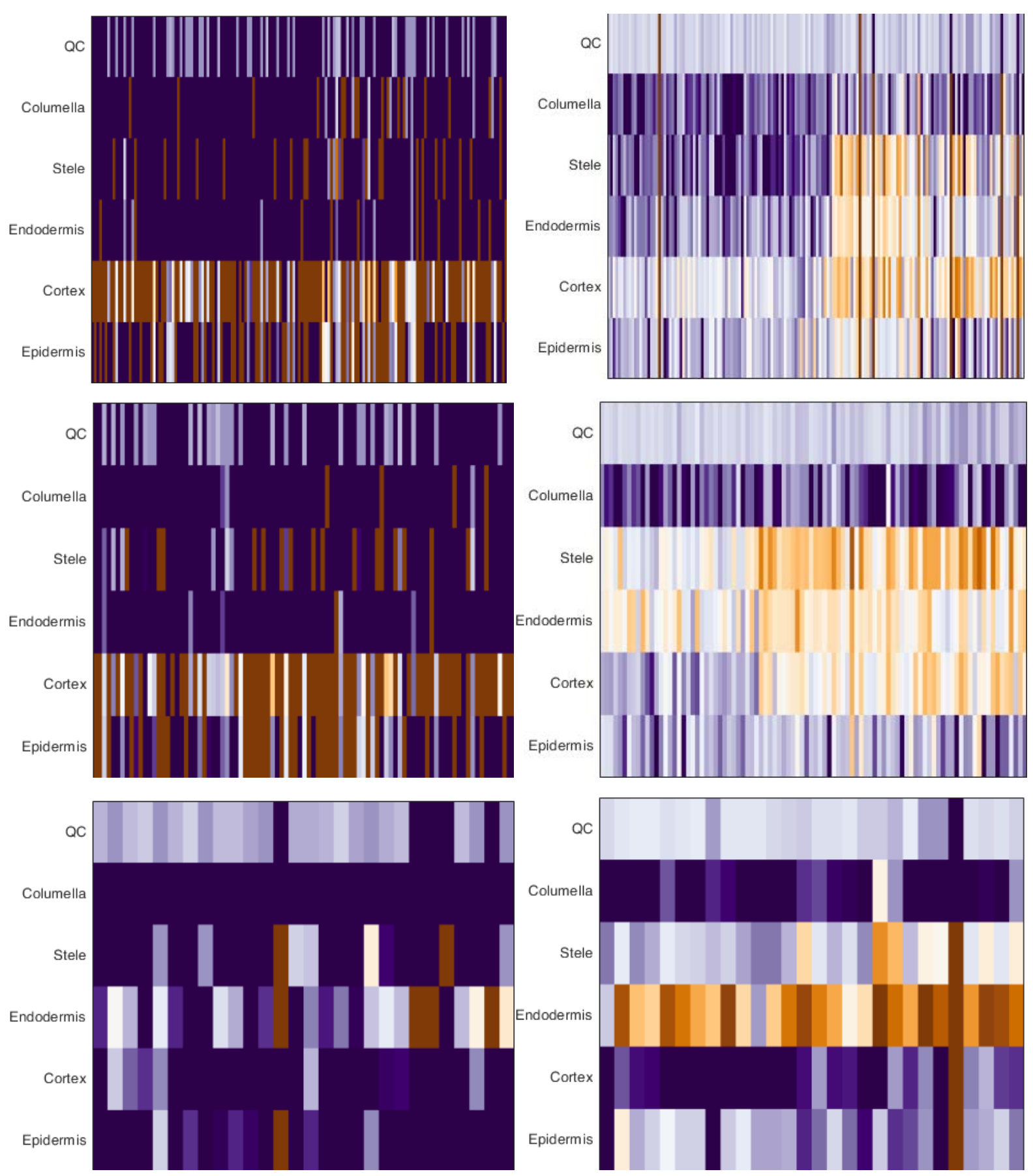

Cluster 15

\section{Fig. S2. ICI analysis of UMAP defined clusters.}

Heatmaps show ICI analysis of each cell (columns) for each cluster. The six different cell types represent the six different tissues profiled by either cell sorting or dissection (root cap or columella). The first column of heatmaps represents an ICI analysis with the 25 best markers for each tissue type (stringent set, see Materials and Methods). The second column of heatmaps represent an ICI analysis with the 150 best markers for each tissue type. When the 25-marker 
analysis gave a weak signal (e.g., cluster 6), the 150-marker analysis was used to narrow down the identification of the cluster in combination with known markers for cell identity (a judgment call given weak quantitative signals). In some rare instances (e.g., cluster 16), the presence of well-documented markers was used to call cell identities over the 150-marker ICI analysis when the stringent 25-marker analysis signal was weak. 
A

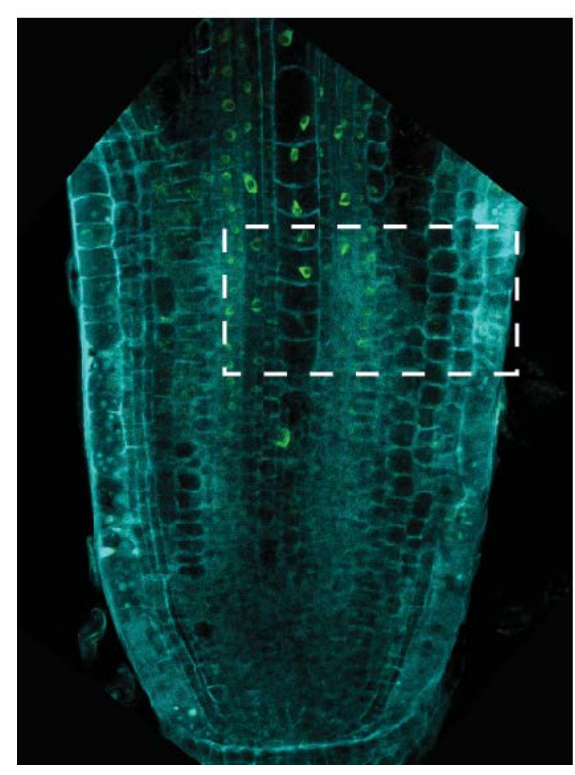

B
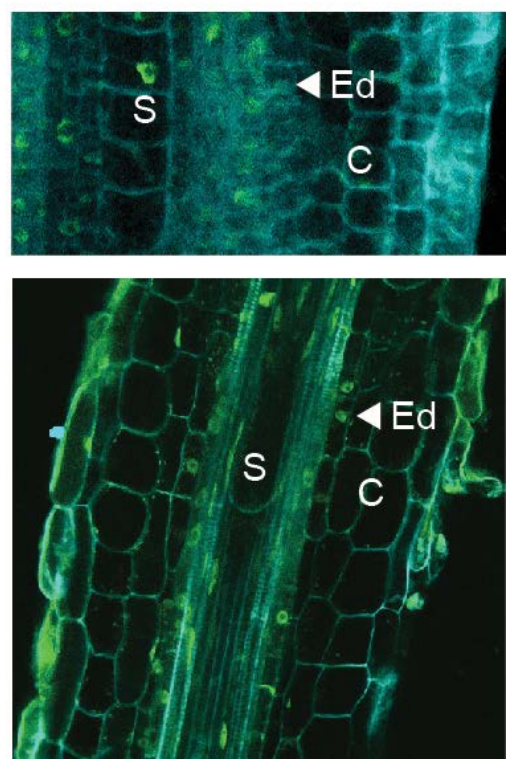

Fig. S3. SCR protein is located in the stele in Setaria viridis roots.

(A) SvSCR translational reporter showing nuclear localization in stele cells. (B) Inset showing enlarged region from dashed box showing endodermis and cortex layers in the root meristem (top), and transition zone (bottom). 
A

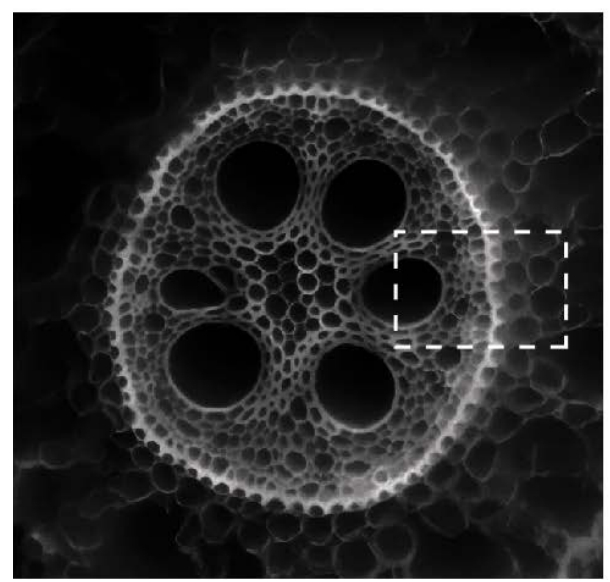

B

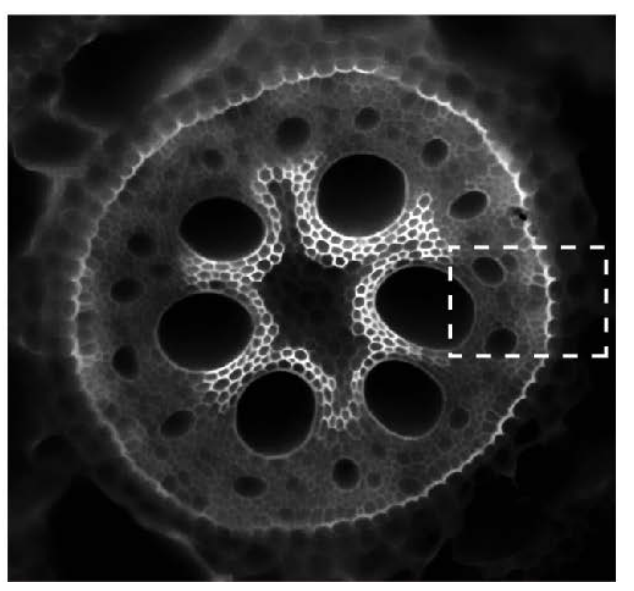

C

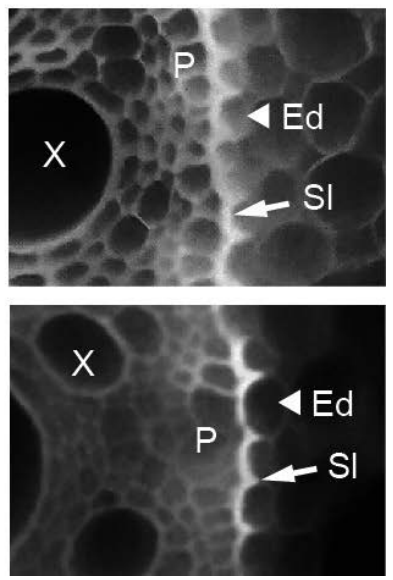

Fig. S4. Endodermal identity analysis by suberin staining in wild type and Zmshr1/3 in maize.

Wild type and Zmshr mutant roots stained with Yellow Fluor 88, which stains the suberin layer (SI) of endodermis (Ed). (A) Cross section of wild type roots showing lens shaped cell with Yellow Fluor88 staining marking an endodermal layer. (B) Cross section of Zmshr2/2-h mutant also showing the lens-shaped cell layer. (C) Magnification of hatched boxes in $C$ and D, showing wild type (top) and Zmshr2/2-h (bottom). Pericycle (P) and xylem vessels (X) can also be observed. 
A

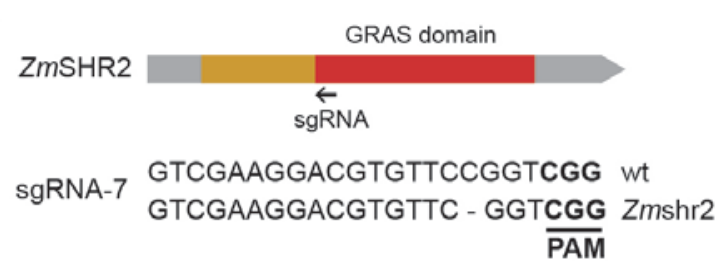

C

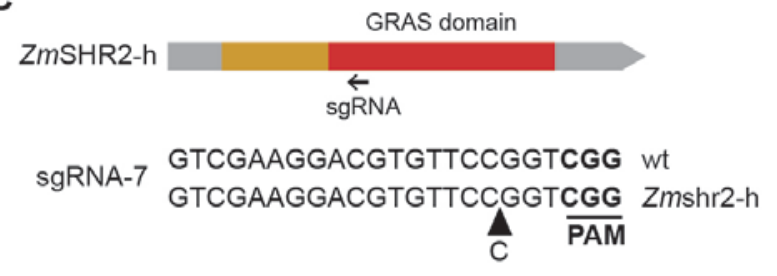

B

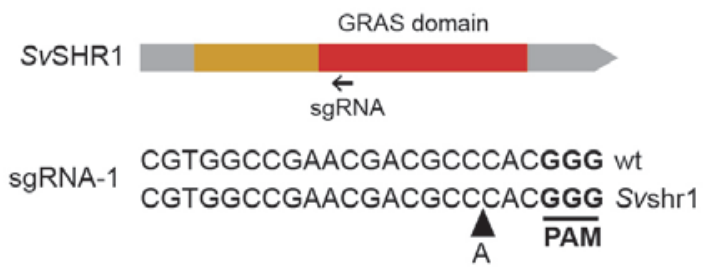

D

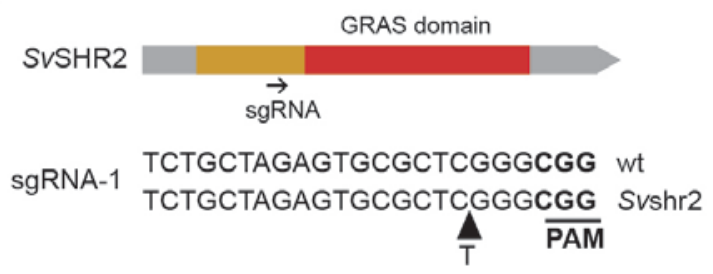

Fig. S5. CRSIPR-Cas9 gene editing in ZmSHR and SvSHR genes caused frameshift mutations.

(A \& C). Diagram showing the position of a single-base deletion in Zmshr2 and a single-based insertion in Zmshr2-h, causing frameshifts. (B \& D). Diagram showing the position of single-base insertions in Svshr1 and Svshr2. Grey box = UTR region, green = exons, red = SHR GRAS domain, arrows $=$ sgRNAs. 